\title{
Preliminary Observations on Sensitivity and Specificity of Magnetization Transfer Asymmetry for Imaging Myelin of Rat Brain at High Field
}

\author{
Jae-Woong Kim, ${ }^{1,2}$ Jiye Choi, ${ }^{1}$ Janggeun Cho, ${ }^{2,3}$ Chulhyun Lee, ${ }^{2}$ \\ Daejong Jeon, ${ }^{1}$ and Sung-Hong Park ${ }^{1}$ \\ ${ }^{1}$ Department of Bio and Brain Engineering, Korea Advanced Institute of Science and Technology, Daejeon 305-701, Republic of Korea \\ ${ }^{2}$ Division of Magnetic Resonance Research, Korea Basic Science Institute, Ochang-eup, Cheongwon-gun, \\ Chungcheongbuk-do 363-883, Republic of Korea \\ ${ }^{3}$ Department of Chemistry, Chung-Ang University, Seoul 156-756, Republic of Korea
}

Correspondence should be addressed to Sung-Hong Park; sunghongpark@kaist.ac.kr

Received 26 February 2015; Revised 15 June 2015; Accepted 8 July 2015

Academic Editor: Gulsum Ozyigit

Copyright (C) 2015 Jae-Woong Kim et al. This is an open access article distributed under the Creative Commons Attribution License, which permits unrestricted use, distribution, and reproduction in any medium, provided the original work is properly cited.

\begin{abstract}
Magnetization transfer ratio (MTR) has been often used for imaging myelination. Despite its high sensitivity, the specificity of MTR to myelination is not high because tissues with no myelin such as muscle can also show high MTR. In this study, we propose a new magnetization transfer (MT) indicator, MT asymmetry (MTA), as a new method of myelin imaging. The experiments were performed on rat brain at 9.4 T. MTA revealed high signals in white matter and significantly low signals in gray matter and muscle, indicating that MTA has higher specificity than MTR. Demyelination and remyelination studies demonstrated that the sensitivity of MTA to myelination was as high as that of MTR. These experimental results indicate that MTA can be a good biomarker for imaging myelination. In addition, MTA images can be efficiently acquired with an interslice MTA method, which may accelerate clinical application of myelin imaging.
\end{abstract}

\section{Introduction}

Myelin is an essential microstructure component for normal brain function. It is a type of glial cells that wraps around axons, forming dielectric myelin sheath structure. Myelin induces saltatory conduction enabling nerve impulse to propagate more than 200 times faster. Moreover, myelin accounts for $50 \%$ of the dry weight of central nervous system [1]. For these reasons, imaging and observing myelination state are important to understand neurological state and diagnose neural diseases. The gold standard to image myelination is direct observation using staining [2]. Various histochemistry methods have been developed and verified by comparing normal and impaired brains. However, the methods are not applicable to clinical studies due to invasiveness. Medical imaging devices, therefore, have drawn neurological and neuropathological interests for noninvasive clinical diagnosis of myelin-related diseases such as multiple sclerosis (MS) [37] and schizophrenia [8-11].

Magnetic resonance imaging (MRI) provides apparent soft tissue contrast and various approaches have been developed for imaging myelin distribution using MRI. Myelin water imaging is an approach that utilizes short $T_{2}$ decay of water in myelin sheath $[12,13]$. The method assumes the measured decay curve to be weighted sum of multiexponential functions. A nonnegative least square algorithm plays a crucial role in converting the decay curves to $T_{2}$ distribution and thus clearly separating myelin water signal from the other tissue water signals [5, 12-17]. However, long scan time per slice and long data processing time hinder its clinical applications. Other approaches for separation of myelin from other components based on faster decay of 
myelin are $T_{2}{ }^{*}$-based methods $[7,18]$ and a double inversion recovery method [19].

Another fast and efficient MRI approach for myelin imaging is magnetization transfer (MT). MT is a phenomenon of magnetization exchange between free water and proton bound to macromolecules. The exchange enables an RF pulse of off-resonance saturation to decrease signals in free water typically used in MR imaging, thereby indirectly providing information of proton bound to macromolecules. MT ratio (MTR) is typically measured for the MT techniques and has been used for appraising abnormality of brain structures, especially those related to myelination. MTR imaging offers noticeable sensitivity on white matter (WM), showing decreased signal on the myelinated regions in multiple sclerosis (MS) patients [4, 6, 20-22] and demyelinated animals [23-26]. The MTR method has advantages of shorter scan time and shorter postprocessing time than myelin water imaging. However, the MTR signal is not exclusively high in myelinated tissue, indicating low specificity. In other words, MTR can be generated by various pathophysiological components. The water concentration in multiple sclerosis lesions is induced by inflammatory activity and affects the MTR signal $[24,27]$. Also, densely packed structure of biological tissue (i.e., skeletal muscle) has plenty of macromolecular bound protons resulting in high MTR signal [28].

In previous studies, the asymmetric MT effects around the water-resonance frequency have been reported [29-31]. Recently, a study proposed a possibility of imaging myelination by using asymmetric chemical exchange saturation transfer effect in the domain of Nuclear Overhauser Effects [32]. Another previous study directly measured extracted myelin in ${ }^{1} \mathrm{H}$ NMR system and reported that myelin exhibits main peak at $3.5 \mathrm{ppm}$ upfield from water-resonance frequency due to the chemical shift [33]. From these results, it is anticipated that taking advantage of the chemical shift as well as the MT strength (rather than the MT strength alone) would be beneficial to image myelination. This notion indicates that MT asymmetry (MTA) may be a better choice than the conventional MTR approach for improving the specificity of the myelin signals, by suppressing the MT signals from the nonmyelin tissues.

The goal of this study is to assess the sensitivity and specificity of the MTA approach for imaging myelination. We hypothesized that MTA imaging would provide better specificity to myelin than conventional MTR. For the MTA and MTR imaging, we used a new method, alternate ascending/descending directional navigation (ALADDIN), which enables us to acquire interslice perfusion-weighted and MTA images simultaneously [34-37], and MTR imaging was also performed through ALADDIN by additionally acquiring MT free images [38]. We investigated the contrast between WM and other brain tissues on various flip angles and compared the results of MTA to those of MTR. We also examined MTA and MTR in normal rats and demyelinated rats through the histogram analysis, which is typically used in the MTR studies $[3,4,39,40]$, in order to investigate the effects of the myelination state on the two MT measures of MTA and MTR.

\section{Materials and Methods}

2.1. Animal Preparation. Total 6 male Sprague-Dawley rats with 8-9 weeks of age were scanned in this study approved by the Institutional Animal Care and Use Committee at the Korea Basic Science Institute. Before MRI scan, rats were initially anesthetized by $5 \%$ isoflurane mixed with a $3: 7$ mixture of pure oxygen and nitrous oxide in a plastic box. The animals were then fixed in a cradle to minimize motion and the isoflurane level was decreased at $1.5 \%$ for anesthesia with a breath mask. The body temperature was maintained at around $37^{\circ} \mathrm{C}$. After being placed inside the MRI system, the animals were monitored for the breath rate to be kept at 50-70 per minute by adjusting the isoflurane level at around $1.5 \%$. To examine demyelination effects, 4 rats $(2$ control and 2 demyelinated rats) with 8 weeks of age were used for the MRI scans. For the 2 rats of demyelination, $0.2 \%$ (w/w) cuprizone (bis-cyclohexanone oxaldihydrazone, Sigma-Aldrich Inc., St. Louis, MO, USA) mixed into a ground standard rodent chow from 4 weeks to 8 weeks of age [41]. In order to study remyelination effects, the cuprizone administration was stopped for the demyelinated rats from 8 weeks of age (the first MRI study) to 9 weeks of age (for one week) and then the second MRI study was performed for the remyelinated rats.

2.2. Data Acquisition. All experiments were conducted on a 9.4 T Varian animal MRI system (Palo Alto, CA, USA) with a volume RF coil (a diameter of $72 \mathrm{~mm}$ ) for both transmission and reception of the signals. All the MTA and MTR acquisitions were performed using ALADDIN based on balanced steady state free precession (bSSFP) readout, as described previously at low fields [34-36, 38]. Briefly, 4 different types of data were acquired by alternating the slice acquisition order (i.e., ascending and descending) and the slice-select gradient polarity (denoted as PosAsc, NegAsc, PosDes, and NegDes in Figure 1). The MTA was acquired by combining the 4 datasets to separate interslice MTA out of the interslice blood flow effects, as described in Figure 1 and also in the references $[34,36]$. The readout gradient polarity was also alternated for averaging to suppress the effects of gradient imperfections, and thus eventually total 8 different types of data were acquired $[34,36]$.

2.2.1. Flip Angle-Dependent Study. To investigate the specificity of magnetization transfer signals of WM relative to the other brain tissues, 2 healthy male rats with 8-9 weeks of age were scanned using ALADDIN at various flip angles of $30^{\circ}, 45^{\circ}$, and $60^{\circ}$. The imaging parameters were $\mathrm{TR}=3.8 \mathrm{~ms}$, $\mathrm{TE}=1.9 \mathrm{~ms}$, matrix size $=128 \times 128$, field of view $=30 \times$ $30 \mathrm{~mm}^{2}$, number of slices $=21$, phase cycle angle $=180^{\circ}$, slice thickness $=1 \mathrm{~mm}$, phase over sampling $=0 \%-100 \%$, and total scan time $=8.2-13.6 \mathrm{~min}$. A large interslice gap $(100 \%$ of the slice thickness) was used to avoid the crosstalk effects. The interslice offset frequency for the first prior slice was $6700 \mathrm{~Hz}$ corresponding to $16.7 \mathrm{ppm}$ at $9.4 \mathrm{~T}$. For MTR imaging, MT free acquisition was performed with interslice delay of 6 

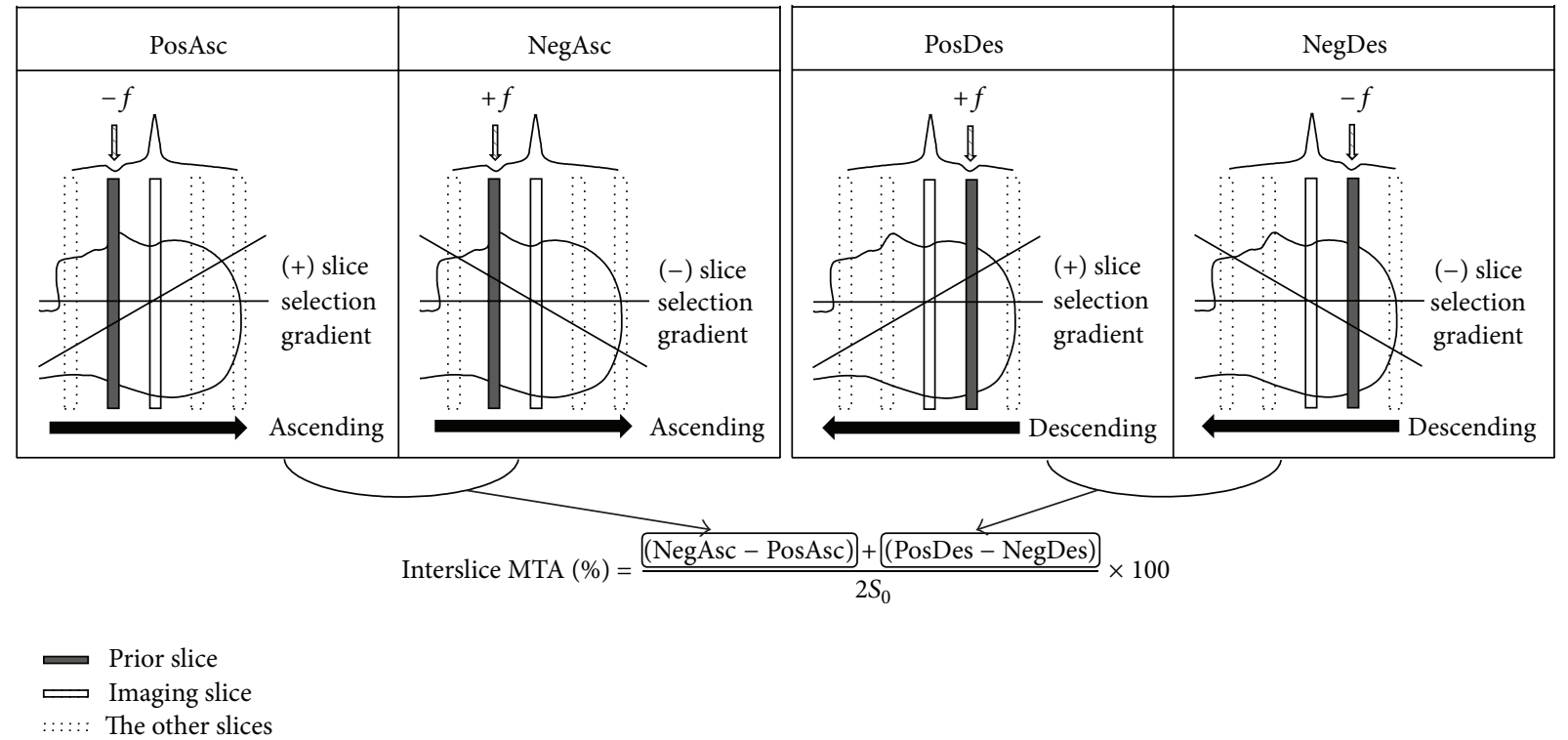

FIGURE 1: Alternate ascending/descending directional navigation (ALADDIN) data acquisition and reconstruction schemes used in this study. Four different types of data acquisition were sequentially performed by alternating the slice acquisition order (ascending and descending, "Asc" and "Des") and the slice-selection gradient polarity (positive and negative, "Pos" and "Neg"). The on-resonance RF pulses for imaging the prior slices simultaneously act as off-resonance saturation pulses for the present imaging slice. $f$ indicates the saturation offset frequency induced by the first prior slice. The four types of images (PosAsc, NegAsc, PosDes, and NegDes) are combined to generate an interslice MT asymmetry (MTA) image. $S_{0}$ represents the average of the four types of images.

seconds for longitudinal magnetizations to recover back to the equilibrium state. The MT free scan was averaged once. MTR images were calculated using the formula: MTR (\%) $=\left(S_{0}-S_{\mathrm{MT}}\right) / S_{0} \times 100$, where $S_{0}$ and $S_{\mathrm{MT}}$ stand for MT free and MT weighted images. $S_{\mathrm{MT}}$ was obtained by averaging the images from both positive and negative offset frequencies.

\subsubsection{Demyelination and Remyelination Effects on MT Signals.}

The two demyelinated rats and the two control rats were scanned at 8 weeks of age. The remyelinated rats were scanned one week after the first MRI scan (i.e., 9 weeks of age). The scan parameters were similar to the above flip angledependent study except TR $=3.8-4.4 \mathrm{~ms}$, TE $=1.9-2.2 \mathrm{~ms}$, bandwidth $=64-100 \mathrm{kHz}$, matrix size $=128 \times 128$, flip angle $=60^{\circ}$, phase oversampling $=0 \%$, and scan time per dataset $=31.1-37.8 \mathrm{~min}$. The ALADDIN acquisitions were averaged 24 times, resulting in the number of repeated acquisitions of volume to be 192. Because of the long scan time, the water-resonance frequency was recalibrated every 3 repeated volume acquisitions to minimize $B_{0}$ drift effects. MT free scan was averaged 4 times with interslice delay of $5 \mathrm{~s}$.

2.3. Data Processing and Analysis. For each rat, we selected a slice of interest that included both corpus callosum (CC) and internal capsule (IC). The regions of interest (ROIs) were manually defined in CC, IC, GM, and muscle areas from each hemisphere using MATLAB, avoiding regions of banding artifacts ( $N=4$ hemispheres for each group). Figure 2 presents the exemplary ROIs and slice of interest in a rat brain. All statistical analyses were performed based

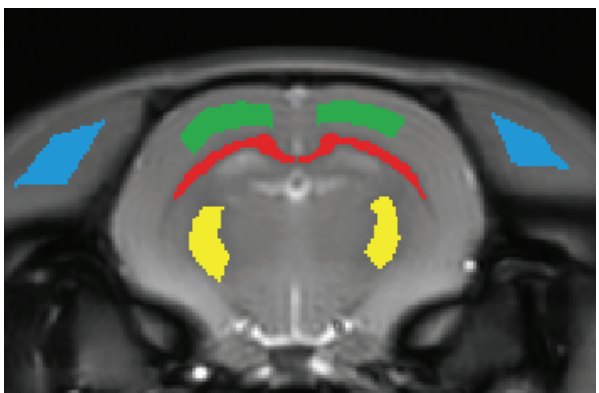

FIGURE 2: Regions of interest (ROIs) manually defined in each hemisphere from a baseline image. Red: corpus callosum (CC), yellow: internal capsule (IC), green: gray matter (GM), and blue: muscle. White matter (WM) ROI is combination of CC and IC.

on the average intensities within the ROIs by using onetailed nonparametric tests at the significance level of $\alpha=0.1$ because of the small sample size.

In the flip angle-dependent study, average intensities were calculated within WM (CC + IC), GM, and muscle ROIs and then plotted as a function of flip angle. Subsequently, specificity was evaluated based on the definition that specificity is the proportion of negatives that are correctly measured. If a technique is prominently sensitive only to WM but not to tissues with no myelination (e.g., muscle), then it is considered having high specificity to myelination. The statistical significance was assessed between WM and GM as well as between WM and muscle on each flip angle by using the Wilcoxon signed rank test. 


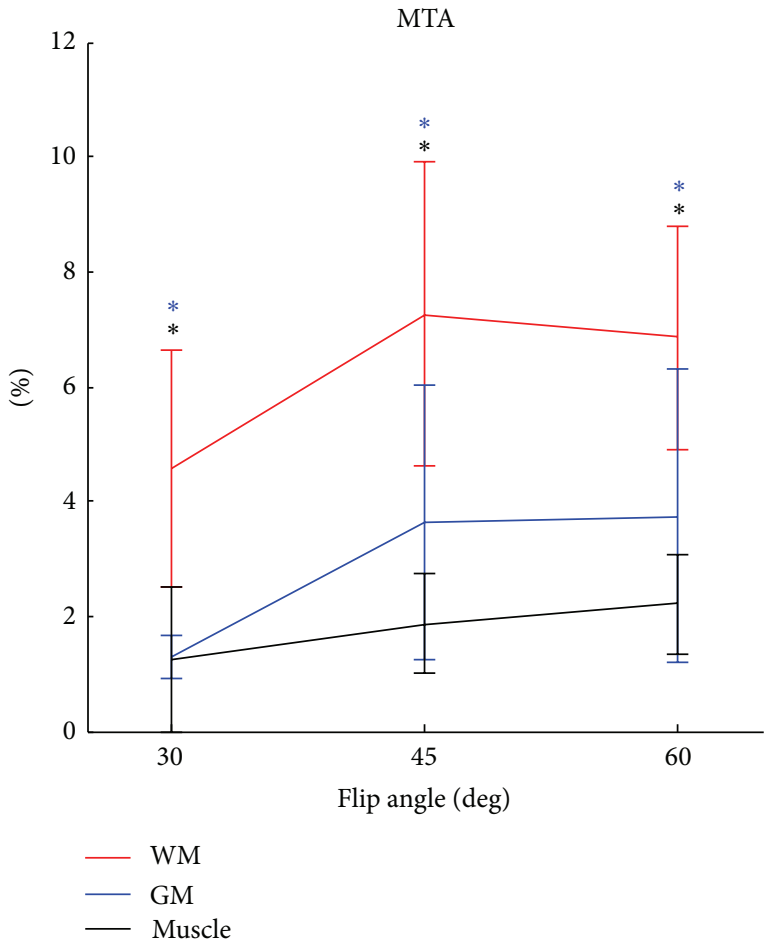

(a)

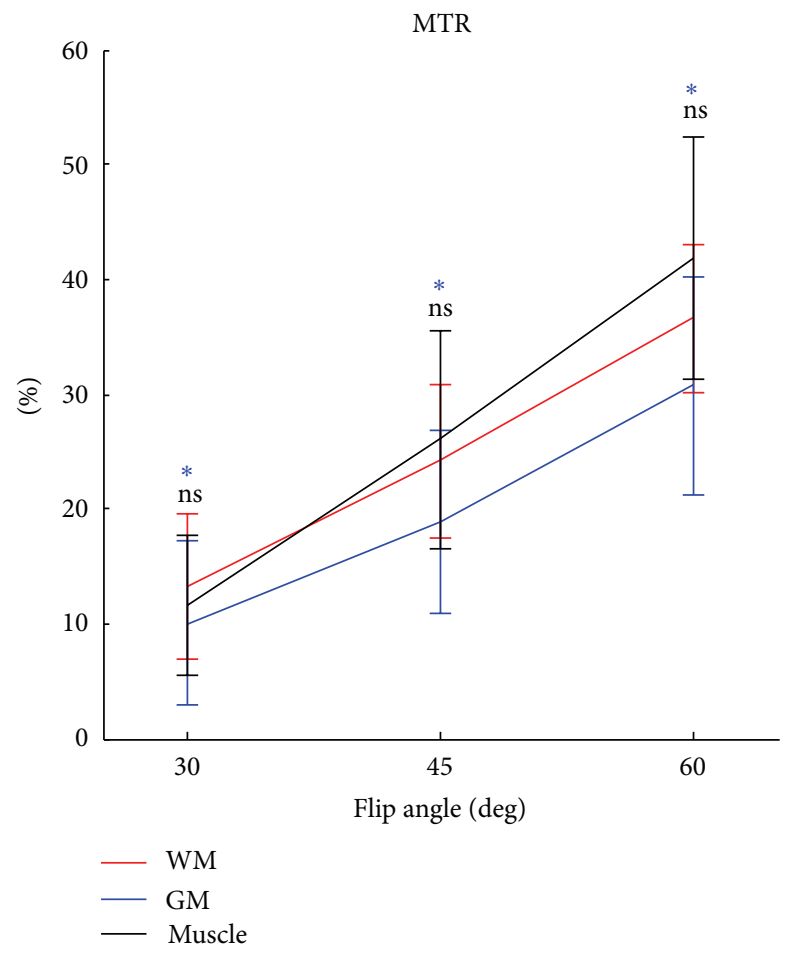

(b)

FIgure 3: MT asymmetry (MTA) and MT ratio (MTR) plots as a function of flip angle. The error bars represent the range of one standard deviation. The blue and black asterisks (upper and lower rows, resp.) represent the significant differences between WM and GM and between WM and muscle, respectively. $*: p<0.1$, ns: $p \geq 0.1$.

In the study of demyelination and remyelination effects on the MT signals, histograms were obtained from the ROIs and normalized by the number of pixels within the ROI. Subsequently, the histograms of MTA and MTR from demyelinated rats and control rats were compared in regard to histogram shape, mean value, and standard deviation within the ROIs. Because most of the MTA and MTR signals were less than $15 \%$ and $45 \%$, respectively, MTA and MTR histograms were plotted within ranges of $0 \%-15 \%$ and $0 \%-$ $45 \%$, respectively, with the same number of bins. Sensitivity was assessed for the demyelination and remyelination study. Sensitivity is the proportion of positives that are correctly measured. If changes in the myelin contents through demyelination and remyelination can be detected, the technique is considered having high sensitivity to myelination. Therefore, the statistical significance was assessed between demyelinated and control rats and between remyelinated and control rats in both WM and GM by using the Wilcoxon rank-sum test.

\section{Results}

3.1. Flip Angle-Dependent Study. Figure 3 shows the MTA and MTR signal intensities, which generally increased with flip angle in most cases. However, there were differences in the results of the ROI analysis between MTA and MTR. The MTA signals showed plateau or even decreased at $60^{\circ}$, whereas the MTR signals continuously increased with flip angle. MTA showed much higher WM signals than GM and muscle, while MTR showed WM signals as high as muscle signals. The statistical tests showed that MTA discriminated WM from the other tissues, but MTR showed difference only between WM and GM (Figure 3), indicating that MTA is more specific to myelinated tissue than MTR. The visual inspection of the MTA and MTR images showed similar results (Figure 4). In the MTR images (the first row in Figure 4), WM signals were strong; however, GM and muscle signals were also not negligible. The MTA images (the second row of Figure 4) also showed higher intensities in WM but noticeably suppressed signals in the other tissue regions.

3.2. Demyelination and Remyelination Effects on MT Signals. Figures 5 and 6, respectively, show histograms from tissue regions of demyelinated and remyelinated rats in comparison with those of control rats. For both MTA and MTR, the histograms of demyelinated rats shifted left compared to those of the control rats, indicating signal decrease due to demyelination effects. In contrast to the results of demyelinated rat brains, the histograms from the remyelinated rats showed almost no difference from those of the control rats in both MTA and MTR (Figure 6), indicating signal recovery due to the remyelination effects.

The quantitative MTA and MTR values for the demyelinated, remyelinated, and control rats (Figure 7) were roughly in agreement with the histogram analysis. Both MTA and 

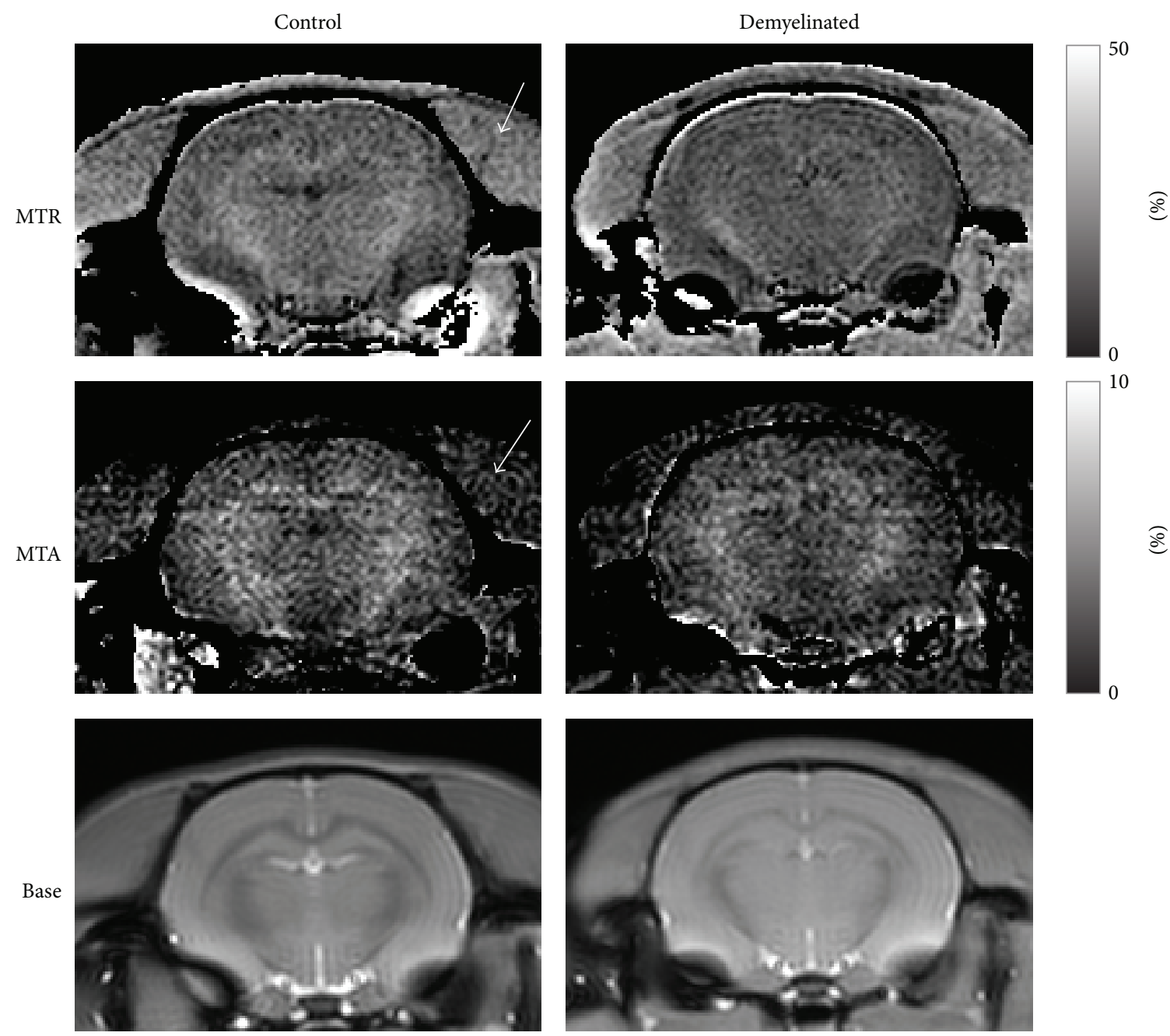

Figure 4: Representative MT asymmetry (MTA), MT ratio (MTR), and baseline (Base) images. Left and right columns correspond to images of control rats and demyelinated rats, respectively. The white arrow indicates the muscle region, where MTA and MTR signals are suppressed and enhanced, respectively.

MTR commonly show signal decrease from demyelination and signal increase from remyelination. However, the statistical results showed that MTA signal changes were significant only for WM whereas MTR signal changes were significant for both WM and GM except GM in the remyelinated rat brain (Figure 7).

\section{Discussion}

The ROI analysis of the flip angle-dependent study and the visual inspection of the representative images indicate that MTA provides better WM specificity than MTR (Figures 3 and 4). Also, the demyelination and remyelination studies indicate that MTA has sensitivity to myelination as high as MTR (Figures 5-7). The MTA signal is derived from asymmetric MT spectrum, indicating that the strength of MT saturation effect is not symmetric around the waterresonance frequency. According to the previous studies, the saturation is stronger at the negative offset frequency $(-f)$ than the positive offset frequency $(+f)$, because of the negatively shifted MT spectrum of macromolecular proton pool in the central nervous system [11, 29-31].

High MTA can be achieved when the two conditions are satisfied: (i) the shift in the center of bound proton pool MT spectrum compared to free proton pool and (ii) strong MT saturation which depends on the tissue type. As shown in the result part, MTA exhibited noticeably high signals in WM and low signals in the other brain tissues. We can postulate that the distinction of WM in MTA method is due to a certain component prevalent in WM, but rare in GM and muscle. In the brain, myelin is a leading candidate for the main signal source of MTA, because myelin satisfies the two conditions above. First, myelin is mostly made up of lipid which forms a main peak centered at about $3.5 \mathrm{ppm}$ upfield from water-resonance frequency in ${ }^{1} \mathrm{H}$ NMR system due to the chemical shift [33]. About $80 \%$ of myelin is lipid and $\sim 20 \%$ is protein [42]. The lipid composition is much higher in WM (49-66\%) than in GM (36-40\%). Therefore, the 


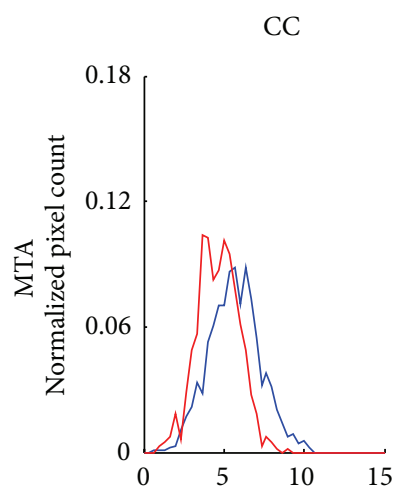

(\%)



(\%)

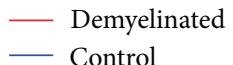

IC

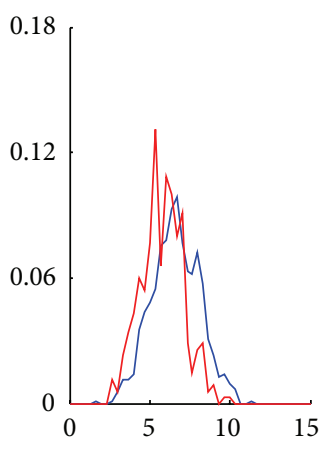

(\%)



(\%)

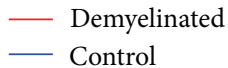

WM



(\%)

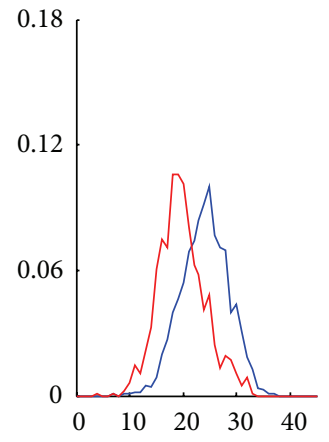

(\%)

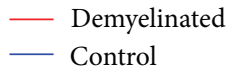

GM

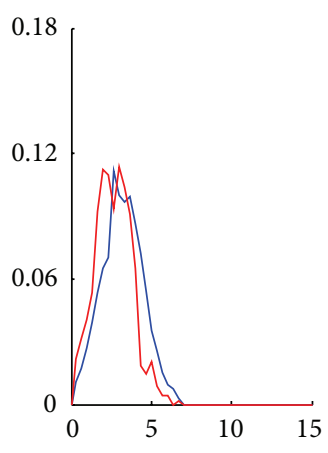

(\%)

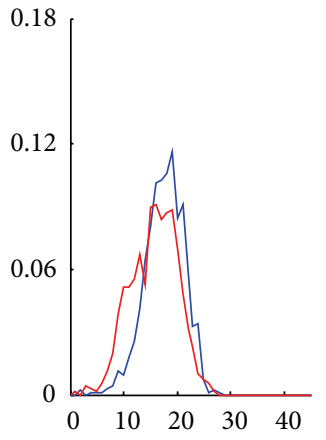

(\%)

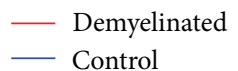

Muscle

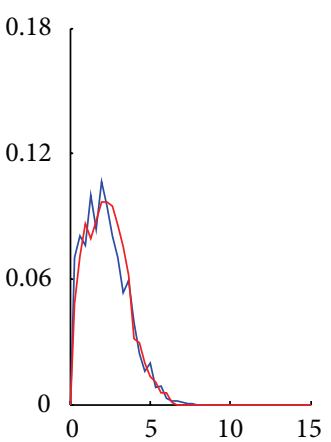

(\%)

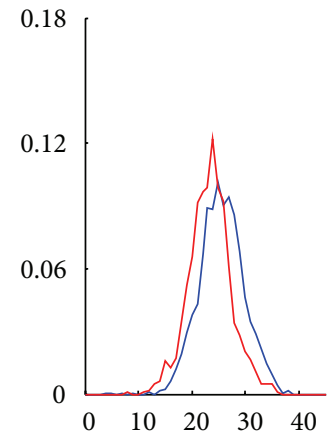

(\%)

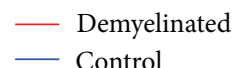

FIGURE 5: Normalized histogram results from demyelinated rats and control rats showing demyelination effects. CC: corpus callosum, IC: internal capsule, WM: white matter (CC + IC), and GM: gray matter.

exchange of magnetizations takes place mostly between water protons and lipid-bound aliphatic protons which compose the side chains of myelin. The interaction between free proton pool and aliphatic proton pool relates to Nuclear Overhauser Effects and provides broad contribution to the MT spectrum around $-3.5 \mathrm{ppm}[32,43]$. Second, from previous studies about MTR, myelin is known to be sensitive to MT-related imaging techniques indicating that myelin has MT effects as high as or even stronger than those in the other brain tissues $[39,44,45]$. As a result, it is plausible that myelin property of the high lipid composition induces not only shift in MT spectrum of bound proton pool but also vast contribution of MT saturation, resulting in high MTA signal.

Sensitivity of MTA to myelination was demonstrated to be similar to that of MTR through the demyelination and remyelination studies (Figures 5-7). MTA successfully detected the change of myelination state caused by the cuprizone administration (Figure 5) and one week interval of no cuprizone administration (Figure 6) in the same manner as MTR, which is in agreement with the other MTR studies [25, $26,46]$. In this study, the MTR signal loss from cuprizoneinduced demyelination was lower than that in other studies $[25,46,47]$ and the MTR recovery from remyelination was relatively faster compared to the results of other studies $[25$,
$26,46]$. It could be due to the fact that the dose of cuprizone in this study $(0.2 \%)$, which has been typically adopted for demyelination of mice rather than rats $[25,26,46,48-$ 50], was relatively lower than the dose used in the other studies for demyelination of rats $[51,52]$. The remyelination effects observed in this study after one week of no cuprizone administration were generally in agreement with the previous studies, which reported significant remyelination shortly after the termination of cuprizone diet $[48,49,53,54]$.

Small signal changes in GM were observed with demyelination and remyelination for MTR (Figure 7). Similar signal changes in GM were also observed for MTA in the histogram analysis, despite being statistically not significant (Figure 7). This could be ascribed to the recovery of small amount of myelin in GM [55]. The oral administration of cuprizone might induce a global demyelination regardless of tissue types. The cuprizone-induced demyelination in cerebral cortex is found in another animal study with decrease of immunodetection [52]. Decreased MTR signal in GM was also reported in previous studies about MS patients [40, 5658]. In a previous study with demyelinated and remyelinated mice [26], the MTR signal changes in deep GM reached statistical significance but those in the cerebral cortex (similar to GM in this study) were not significant, an observation 


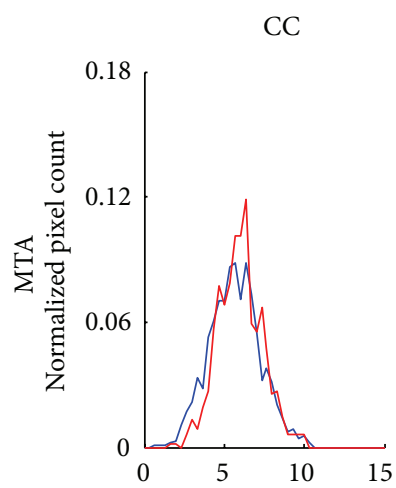

(\%)

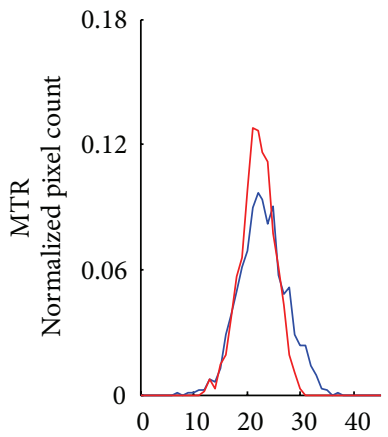

(\%)

- Remyelinated

- Control
IC

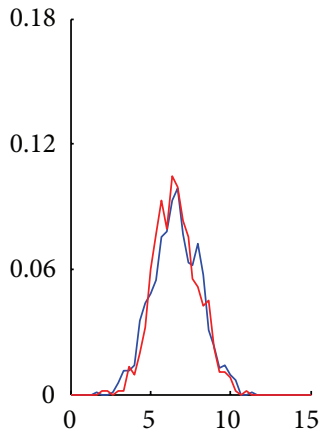

(\%)

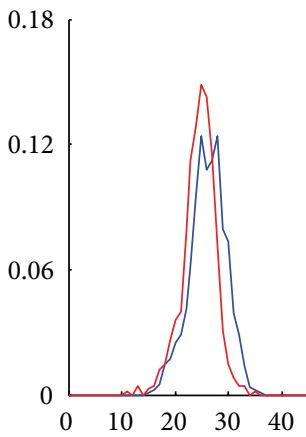

(\%)

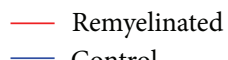

WM

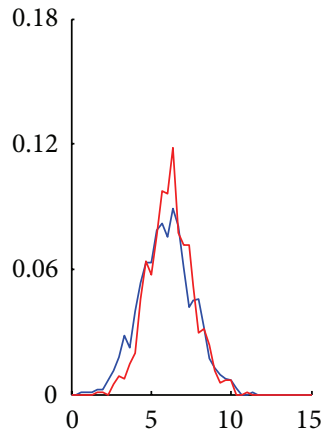

(\%)

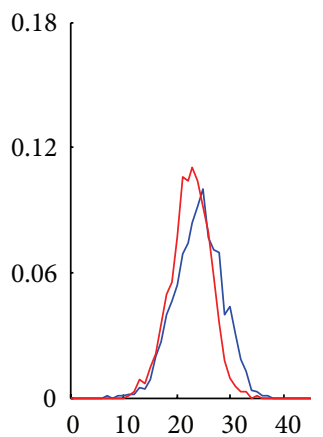

(\%)

- Remyelinated
GM



(\%)

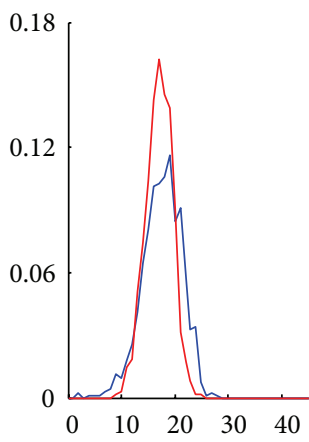

(\%)



Muscle

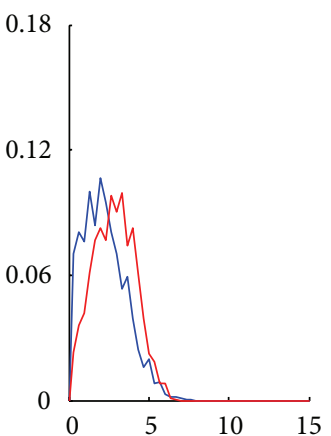

(\%)

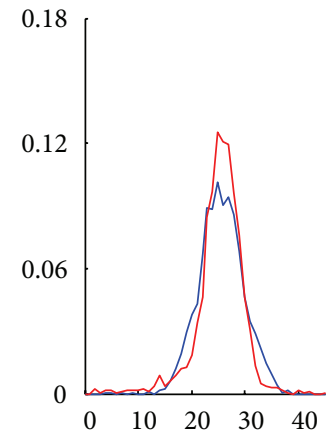

(\%)

- Remyelinated

FIGURE 6: Normalized histogram results from remyelinated rats and control rats showing remyelination effects. CC: corpus callosum, IC: internal capsule, WM: white matter (CC + IC), and GM: gray matter.

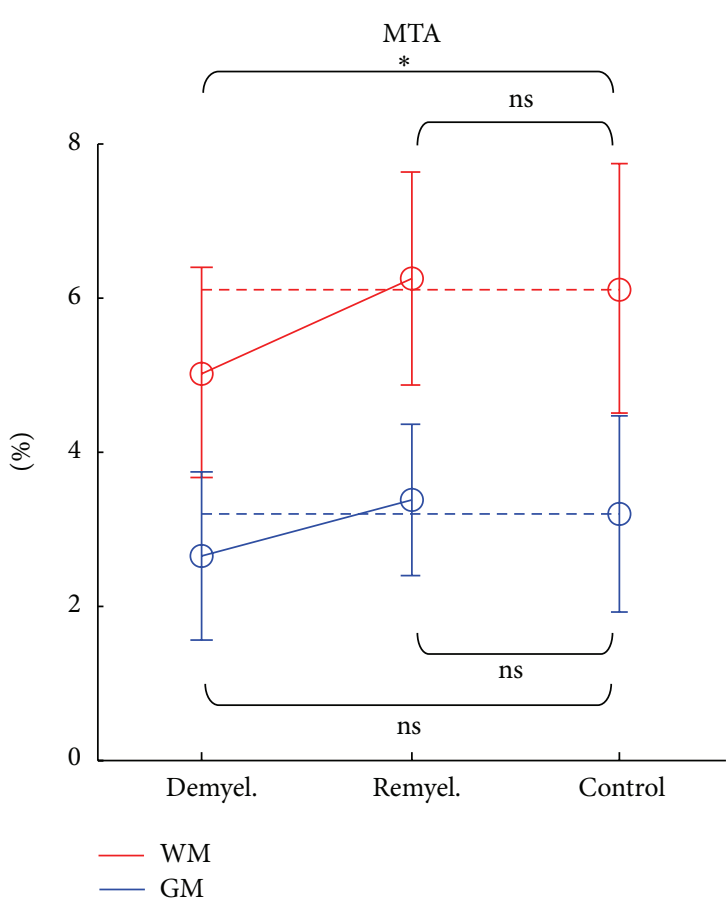

(a)



(b)

Figure 7: MT asymmetry (MTA) (a) and MT ratio (MTR) (b) values for demyelinated (Demyel.), remyelinated (Remyel.), and control rats. MTA and MTR are plotted in different intensity scales. WM: white matter, GM: gray matter. $*: p<0.1, \mathrm{~ns}: p \geq 0.1$. 
slightly different from this study. Therefore, further studies are necessary to assess the significance of signal changes in GM associated with demyelination and remyelination.

The high MTR signal from muscle may be attributed to the unique structure of the muscle tissue. Muscle has numerous micromuscle fibers aligned in parallel that are surrounded by electrolyte, providing a suitable environment for transfer of magnetization. However, unlike the case of myelin, the MT effect does not mainly occur between lipid proton pool and free proton pool, resulting in mostly symmetric MT spectrum and thus low MTA signals.

When bSSFP readout is combined to interslice imaging method based on ALADDIN, the scan time can be effectively reduced because of the absence of MT presaturation period, which was already demonstrated in the clinical scanner [34, 36]. This advantage of ALADDIN may potentially facilitate clinical application of MTA in a reasonable scan time.

\section{Conclusion}

In this study, MTA was compared to conventional myelin imaging technique, MTR, in terms of imaging myelination. MTA imaging revealed high signal in WM and significantly low signals in GM and muscle, indicating better specificity than MTR. The demyelinated rats revealed apparent decrease of MTA and MTR signals, whereas remyelinated rats showed enhanced MTA and MTR signals comparable to those of normal rats. The main signal source of MTA in WM is presumed to be the MT effects occurring between free proton pool and lipid-bound proton pool associated with myelination. The experimental results indicate that MTA can be a good biomarker for imaging myelination with better specificity than and similar sensitivity to MTR. In addition, MTA images can be efficiently acquired with ALADDIN, which may accelerate clinical application of myelination imaging.

\section{Conflict of Interests}

The authors declare that there is no conflict of interests regarding the publication of this paper.

\section{Acknowledgments}

This work was supported by National Research Foundation of Korea (NRF-2013R1A1A1061759) and the Basic Science Research Program Grant (NRF-2014R1A2A2A01002608) funded by Ministry of Science, ICT and Future Planning.

\section{References}

[1] N. Baumann and D. Pham-Dinh, "Biology of oligodendrocyte and myelin in the mammalian central nervous system," Physiological Reviews, vol. 81, no. 2, pp. 871-927, 2001.

[2] H. Klüver and E. Barrera, "A method for the combined staining of cells and fibers in the nervous system," Journal of Neuropathology \& Experimental Neurology, vol. 12, no. 4, pp. 400403, 1953.
[3] J. L. Ostuni, N. D. Richert, B. K. Lewis, and J. A. Frank, "Characterization of differences between multiple sclerosis and normal brain: a global magnetization transfer application," American Journal of Neuroradiology, vol. 20, no. 3, pp. 501-507, 1999.

[4] I. Catalaa, R. I. Grossman, D. L. Kolson et al., "Multiple sclerosis: magnetization transfer histogram analysis of segmented normal-appearing white matter," Radiology, vol. 216, no. 2, pp. 351-355, 2000.

[5] C. Laule, I. M. Vavasour, G. R. Moore et al., "Water content and myelin water fraction in multiple sclerosis. A T2 relaxation study," Journal of Neurology, vol. 251, no. 3, pp. 284-293, 2004.

[6] K. Schmierer, F. Scaravilli, D. R. Altmann, G. J. Barker, and D. H. Miller, "Magnetization transfer ratio and myelin in postmortem multiple sclerosis brain," Annals of Neurology, vol. 56, no. 3, pp. 407-415, 2004.

[7] Y. P. Du, R. Chu, D. Hwang et al., "Fast multislice mapping of the myelin water fraction using multicompartment analysis of T2* decay at 3T: a preliminary postmortem study," Magnetic Resonance in Medicine, vol. 58, no. 5, pp. 865-870, 2007.

[8] K. O. Lim, M. Hedehus, M. Moseley, A. de Crespigny, E. V. Sullivan, and A. Pfefferbaum, "Compromised white matter tract integrity in schizophrenia inferred from diffusion tensor imaging," Archives of General Psychiatry, vol. 56, no. 4, pp. 367374, 1999.

[9] J. Foong, M. Maier, G. J. Barker, S. Brocklehurst, D. H. Miller, and M. A. Ron, "In vivo investigation of white matter pathology in schizophrenia with magnetisation transfer imaging," Journal of Neurology Neurosurgery \& Psychiatry, vol. 68, no. 1, pp. 70-74, 2000.

[10] S. W. Flynn, D. J. Lang, A. L. Mackay et al., "Abnormalities of myelination in schizophrenia detected in vivo with MRI, and post-mortem with analysis of oligodendrocyte proteins," Molecular Psychiatry, vol. 8, no. 9, pp. 811-820, 2003.

[11] F. Du, A. J. Cooper, T. Thida, A. K. Shinn, B. M. Cohen, and D. Öngür, "Myelin and axon abnormalities in schizophrenia measured with magnetic resonance imaging techniques," Biological Psychiatry, vol. 74, no. 6, pp. 451-457, 2013.

[12] A. MacKay, K. Whittall, J. Adler, D. Li, D. Paty, and D. Graeb, "In vivo visualization of myelin water in brain by magnetic resonance," Magnetic Resonance in Medicine, vol. 31, no. 6, pp. 673-677, 1994

[13] K. P. Whittall, A. L. MacKay, D. A. Graeb, R. A. Nugent, D. K. B. $\mathrm{Li}$, and D. W. Paty, "In vivo measurement of T2 distributions and water contents in normal human brain," Magnetic Resonance in Medicine, vol. 37, no. 1, pp. 34-43, 1997.

[14] S. D. Wolff and R. S. Balaban, "Magnetization transfer contrast (MTC) and tissue water proton relaxation in vivo," Magnetic Resonance in Medicine, vol. 10, no. 1, pp. 135-144, 1989.

[15] A. MacKay, C. Laule, I. Vavasour, T. Bjarnason, S. Kolind, and B. Mädler, "Insights into brain microstructure from the $\mathrm{T}_{2}$ distribution," Magnetic Resonance Imaging, vol. 24, no. 4, pp. 515-525, 2006.

[16] C. Laule, P. Kozlowski, E. Leung, D. K. B. Li, A. L. MacKay, and G. R. W. Moore, "Myelin water imaging of multiple sclerosis at 7 T: correlations with histopathology," NeuroImage, vol. 40, no. 4, pp. 1575-1580, 2008.

[17] S. H. Kolind, B. Mädler, S. Fischer, D. K. B. Li, and A. L. MacKay, "Myelin water imaging: implementation and development at 3.0T and comparison to 1.5T measurements," Magnetic Resonance in Medicine, vol. 62, no. 1, pp. 106-115, 2009. 
[18] D. Hwang, D.-H. Kim, and Y. P. Du, "In vivo multi-slice mapping of myelin water content using $\mathrm{T}^{*}$ decay," NeuroImage, vol. 52, no. 1, pp. 198-204, 2010.

[19] S.-H. Oh, M. Bilello, M. Schindler, C. E. Markowitz, J. A. Detre, and J. Lee, "Direct visualization of short transverse relaxation time component (ViSTa)," NeuroImage, vol. 83, pp. 485-492, 2013.

[20] L. A. Loevner, R. I. Grossman, J. A. Cohen, F. J. Lexa, D. Kessler, and D. L. Kolson, "Microscopic disease in normalappearing white matter on conventional MR images in patients with multiple sclerosis: assessment with magnetization-transfer measurements," Radiology, vol. 196, no. 2, pp. 511-515, 1995.

[21] G. B. Pike, N. De Stefano, S. Narayanan et al., "Multiple sclerosis: magnetization transfer MR imaging of white matter before lesion appearance on T2-weighted images," Radiology, vol. 215, no. 3, pp. 824-830, 2000.

[22] S. Ropele, S. Strasser-Fuchs, M. Augustin et al., "A comparison of magnetization transfer ratio, magnetization transfer rate, and the native relaxation time of water protons related to relapsing-remitting multiple sclerosis," The American Journal of Neuroradiology, vol. 21, no. 10, pp. 1885-1891, 2000.

[23] M. S. A. Deloire-Grassin, B. Brochet, B. Quesson et al., "In vivo evaluation of remyelination in rat brain by magnetization transfer imaging," Journal of the Neurological Sciences, vol. 178, no. 1, pp. 10-16, 2000.

[24] P. J. Gareau, B. K. Rutt, S. J. Karlik, and J. R. Mitchell, "Magnetization transfer and multicomponent T2 relaxation measurements with histopathologic correlation in an experimental model of MS," Journal of Magnetic Resonance Imaging, vol. 11, no. 6, pp. 586-595, 2000.

[25] W. Zaaraoui, M. Deloire, M. Merle et al., "Monitoring demyelination and remyelination by magnetization transfer imaging in the mouse brain at 9.4 T," Magnetic Resonance Materials in Physics, Biology and Medicine, vol. 21, no. 5, pp. 357-362, 2008.

[26] S. Fjær, L. Bø, A. Lundervold et al., "Deep gray matter demyelination detected by magnetization transfer ratio in the cuprizone model," PLoS ONE, vol. 8, no. 12, Article ID e84162, 2013.

[27] I. M. Vavasour, C. Laule, D. K. B. Li, A. L. Traboulsee, and A. L. MacKay, "Is the magnetization transfer ratio a marker for myelin in multiple sclerosis?" Journal of Magnetic Resonance Imaging, vol. 33, no. 3, pp. 710-718, 2011.

[28] D. M. Yousem, M. D. Schnall, L. Dougherty, G. S. Weinstein, and R. E. Hayden, "Magnetization transfer imaging of the head and neck: normative data," American Journal of Neuroradiology, vol. 15, no. 6, pp. 1117-1121, 1994.

[29] J. Pekar, P. Jezzard, D. A. Roberts, J. S. Leigh Jr., J. A. Frank, and A. G. McLaughlin, "Perfusion imaging with compensation for asymmetric magnetization transfer effects," Magnetic Resonance in Medicine, vol. 35, no. 1, pp. 70-79, 1996.

[30] J. Hua, C. K. Jones, J. Blakeley, S. A. Smith, P. C. M. van Zijl, and J. Zhou, "Quantitative description of the asymmetry in magnetization transfer effects around the water resonance in the human brain," Magnetic Resonance in Medicine, vol. 58, no. 4, pp. 786-793, 2007.

[31] M.-C. Ng, J. Hua, Y. Hu, K. D. Luk, and E. Y. Lam, "Magnetization transfer (MT) asymmetry around the water resonance in human cervical spinal cord," Journal of Magnetic Resonance Imaging, vol. 29, no. 3, pp. 523-528, 2009.

[32] O. Mougin, M. Clemence, A. Peters, A. Pitiot, and P. Gowland, "High-resolution imaging of magnetisation transfer and nuclear Overhauser effect in the human visual cortex at 7 T," NMR in Biomedicine, vol. 26, no. 11, pp. 1508-1517, 2013.
[33] M. J. Wilhelm, H. H. Ong, S. L. Wehrli et al., "Direct magnetic resonance detection of myelin and prospects for quantitative imaging of myelin density," Proceedings of the National Academy of Sciences of the United States of America, vol. 109, no. 24, pp. 9605-9610, 2012.

[34] S.-H. Park and T. Q. Duong, "Alternate ascending/descending directional navigation approach for imaging magnetization transfer asymmetry," Magnetic Resonance in Medicine, vol. 65, no. 6, pp. 1702-1710, 2011.

[35] S.-H. Park and T. Q. Duong, "Brain MR perfusion-weighted imaging with alternate ascending/descending directional navigation," Magnetic Resonance in Medicine, vol. 65, no. 6, pp. 15781591, 2011.

[36] S.-H. Park, T. Zhao, J.-H. Kim, F. E. Boada, and K. T. Bae, "Suppression of effects of gradient imperfections on imaging with alternate ascending/descending directional navigation," Magnetic Resonance in Medicine, vol. 68, no. 5, pp. 1600-1606, 2012.

[37] S. H. Park, P. K. Han, and S. H. Choi, "Physiological and functional magnetic resonance imaging using balanced steadystate free precession," Korean Journal of Radiology, vol. 16, no. 3, pp. 550-559, 2015.

[38] J. W. Barker, P. K. Han, S. H. Choi, K. T. Bae, and S.-H. Park, "Investigation of inter-slice magnetization transfer effects as a new method for MTR imaging of the human brain," PLoS ONE, vol. 10, no. 2, Article ID e0117101, 2015.

[39] M. A. van Buchem, J. C. McGowan, D. L. Kolson, M. Polansky, and R. I. Grossman, "Quantitative volumetric magnetization transfer analysis in multiple sclerosis: estimation of macroscopic and microscopic disease burden," Magnetic Resonance in Medicine, vol. 36, no. 4, pp. 632-636, 1996.

[40] L. K. Fisniku, D. R. Altmann, M. Cercignani et al., "Magnetization transfer ratio abnormalities reflect clinically relevant grey matter damage in multiple sclerosis," Multiple Sclerosis, vol. 15, no. 6, pp. 668-677, 2009.

[41] J. R. Gregg, N. R. Herring, A. V. Naydenov, R. P. Hanlin, and C. Konradi, "Downregulation of oligodendrocyte transcripts is associated with impaired prefrontal cortex function in rats," Schizophrenia Research, vol. 113, no. 2-3, pp. 277-287, 2009.

[42] J. S. O'Brien and E. L. Sampson, "Lipid composition of the normal human brain: gray matter, white matter, and myelin," Journal of Lipid Research, vol. 6, no. 4, pp. 537-544, 1965.

[43] D. Liu, J. Zhou, R. Xue, Z. Zuo, J. An, and D. J. J. Wang, “Quantitative characterization of nuclear overhauser enhancement and amide proton transfer effects in the human brain at 7 tesla," Magnetic Resonance in Medicine, vol. 70, no. 4, pp. 1070-1081, 2013.

[44] R. I. Grossman, "Magnetization transfer in multiple sclerosis," Annals of Neurology, vol. 36, supplement 1, pp. S97-S99, 1994.

[45] M. A. Van Buchem, S. C. A. Steens, H. A. Vrooman et al., "Global estimation of myelination in the developing brain on the basis of magnetization transfer imaging: a preliminary study," American Journal of Neuroradiology, vol. 22, no. 4, pp. 762-766, 2001.

[46] D. Merkler, S. Boretius, C. Stadelmann et al., "Multicontrast MRI of remyelination in the central nervous system," NMR in Biomedicine, vol. 18, no. 6, pp. 395-403, 2005.

[47] J. D. Thiessen, Y. Zhang, H. Zhang et al., "Quantitative MRI and ultrastructural examination of the cuprizone mouse model of demyelination," NMR in Biomedicine, vol. 26, no. 11, pp. 15621581, 2013.

[48] M. Lindner, J. Fokuhl, F. Linsmeier, C. Trebst, and M. Stangel, "Chronic toxic demyelination in the central nervous system 
leads to axonal damage despite remyelination," Neuroscience Letters, vol. 453, no. 2, pp. 120-125, 2009.

[49] P. Morell, C. V. Barrett, J. L. Mason et al., "Gene expression in brain during cuprizone-induced demyelination and remyelination," Molecular and Cellular Neurosciences, vol. 12, no. 4-5, pp. 220-227, 1998.

[50] Ø. Torkildsen, L. A. Brunborg, K.-M. Myhr, and L. Bø, "The cuprizone model for demyelination," Acta Neurologica Scandinavica, vol. 117, no. 188, pp. 72-76, 2008.

[51] P. G. Franco, L. Silvestroff, E. F. Soto, and J. M. Pasquini, "Thyroid hormones promote differentiation of oligodendrocyte progenitor cells and improve remyelination after cuprizoneinduced demyelination," Experimental Neurology, vol. 212, no. 2, pp. 458-467, 2008.

[52] L. Silvestroff, S. Bartucci, J. Pasquini, and P. Franco, "Cuprizoneinduced demyelination in the rat cerebral cortex and thyroid hormone effects on cortical remyelination," Experimental Neurology, vol. 235, no. 1, pp. 357-367, 2012.

[53] A. M. Adamo, P. M. Paez, O. E. Escobar Cabrera et al., "Remyelination after cuprizone-induced demyelination in the rat is stimulated by apotransferrin," Experimental Neurology, vol. 198, no. 2, pp. 519-529, 2006.

[54] G. K. Matsushima and P. Morell, "The neurotoxicant, cuprizone, as a model to study demyelination and remyelination in the central nervous system," Brain Pathology, vol. 11, no. 1, pp. 107116, 2001.

[55] K. Suzuki, J. F. Poduslo, and S. E. Poduslo, "Further evidence for a specific ganglioside fraction closely associated with myelin," Biochimica et Biophysica Acta-Lipids and Lipid Metabolism, vol. 152, no. 3, pp. 576-586, 1968.

[56] B. Audoin, G. Davies, W. Rashid, L. Fisniku, A. J. Thompson, and D. H. Miller, "Voxel-based analysis of grey matter magnetization transfer ratio maps in early relapsing remitting multiple sclerosis," Multiple Sclerosis, vol. 13, no. 4, pp. 483-489, 2007.

[57] Y. Ge, R. I. Grossman, J. K. Udupa, J. S. Babb, D. L. Kolson, and J. C. McGowan, "Magnetization transfer ratio histogram analysis of gray matter in relapsing-remitting multiple sclerosis," American Journal of Neuroradiology, vol. 22, no. 3, pp. 470-475, 2001.

[58] Y. Ge, R. I. Grossman, J. K. Udupa, J. S. Babb, L. J. Mannon, and J. C. McGowan, "Magnetization transfer ratio histogram analysis of normal-appearing gray matter and normal-appearing white matter in multiple sclerosis," Journal of Computer Assisted Tomography, vol. 26, no. 1, pp. 62-68, 2002. 

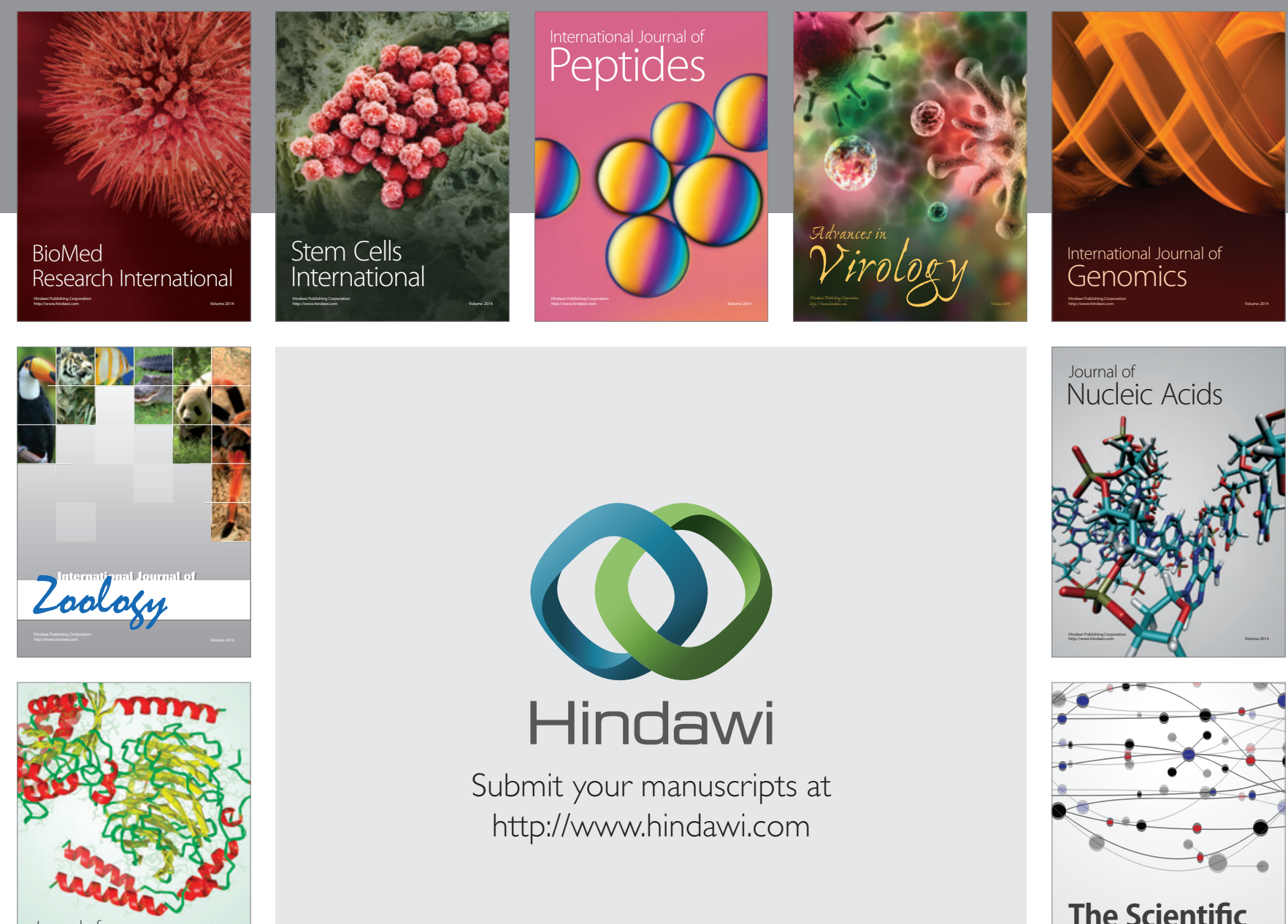

Submit your manuscripts at

http://www.hindawi.com

Journal of
Signal Transduction
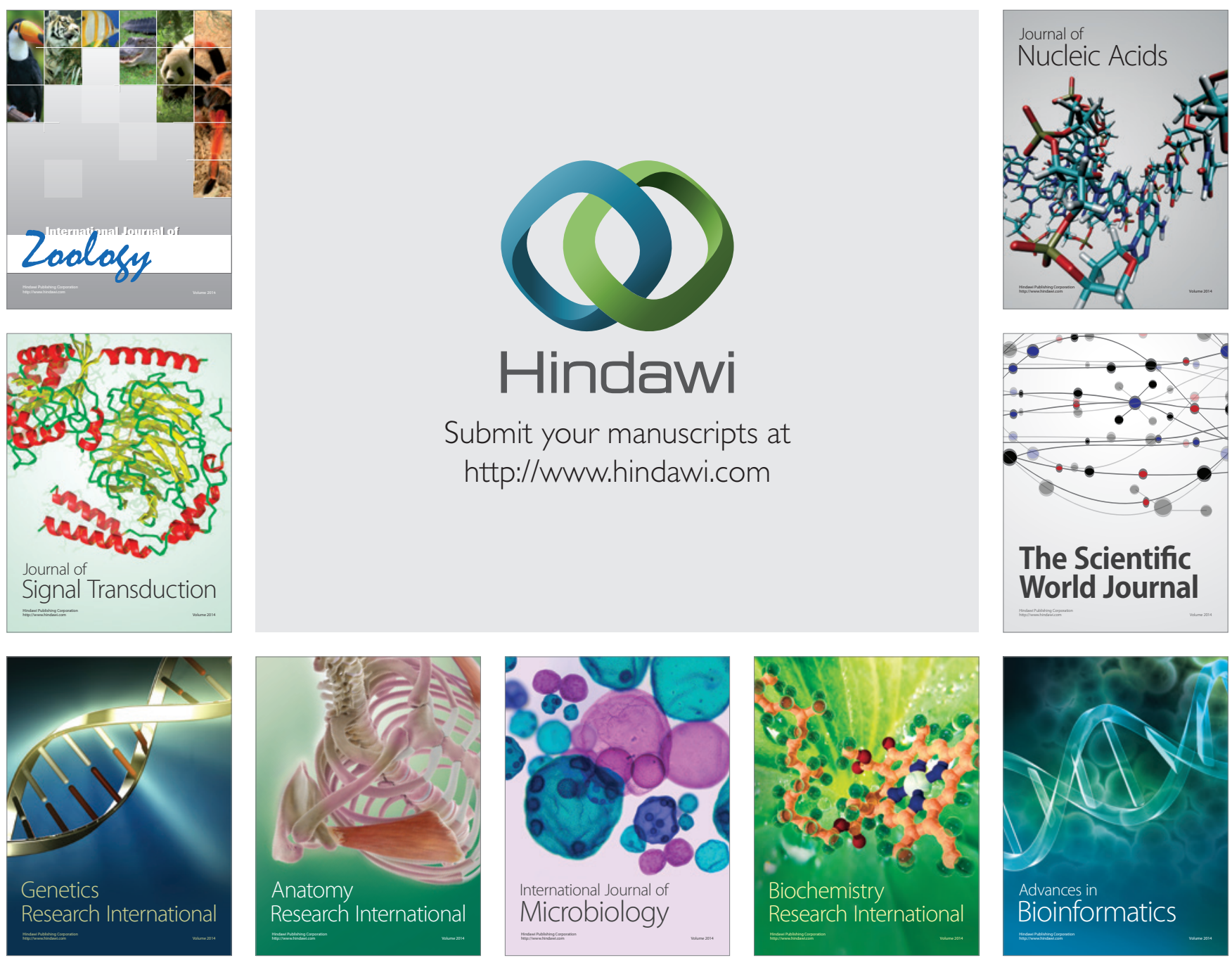

The Scientific World Journal
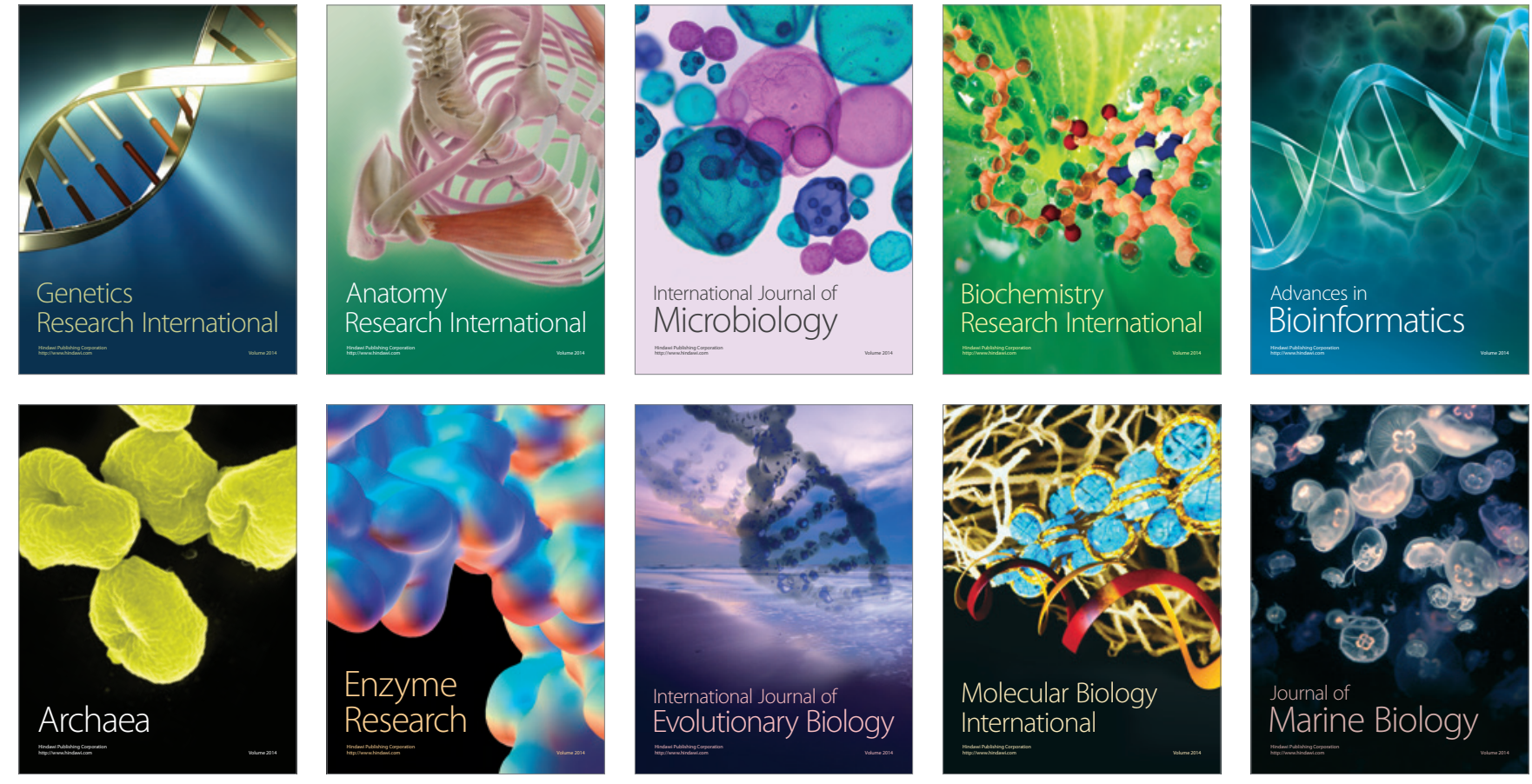